Laís Prado Campos ${ }^{1}$

(iD) Lina Monteiro de Castro Lobo ${ }^{1}$

1 Pontifícia Universidade Católica de Goiás, Curso de Nutrição. Goiânia, GO, Brasil.

Correspondência

Lais Prado Campos

Ipradocampos@gmail.com

\section{Efeitos da restrição de carboidratos no manejo do diabetes mellitus: revisão de literatura científica}

\author{
Effects of carbohydrate-restricted diets in the management of \\ diabetes mellitus: review of scientific literature
}

\begin{abstract}
Resumo
O objetivo do presente estudo foi avaliar, por meio de uma revisão da literatura, evidências da utilização de dietas com restrição de carboidratos no manejo do diabetes mellitus (DM). As buscas eletrônicas e manuais foram até abril de 2019, e incluíram trabalhos publicados a partir de 2008. Foram excluídos estudos de pesquisas in vitro e em animais, revisões de literatura, livros, monografias, dissertações, teses, estudos de caso e relatos de caso. Os artigos remanescentes foram submetidos à análise de sua qualidade metodológica pela Escala JADAD cinco pontos. Um total de 19 estudos randomizados e com qualidade média de três pontos foram selecionados e analisados quanto aos tipos de dieta utilizadas, adesão, tipo de DM, tempo de intervenção, consumo reportado de carboidratos e resultados observados para os grupos controle e intervenção. Dentre os parâmetros escolhidos para mensurar os possíveis efeitos das dietas, destacaram-se alteração de peso e IMC, Hb1Ac, variabilidade ou controle glicêmico, perfil lipídico e alteração nas doses de insulina ou medicação utilizadas. Em quatro estudos não foram observadas vantagens significativas de uma dieta restrita em carboidratos, e 15 trabalhos relataram melhoras em um ou mais parâmetros. Tais resultados podem representar uma grande vantagem na adoção desta estratégia alimentar no manejo do DM e na prevenção de complicações da doença. Contudo, existem limitações nos estudos, que precisam ter suas hipóteses verificadas no longo prazo, e pesquisas adicionais devem ser realizadas para configurar uma estratégia oficial no controle do DM.
\end{abstract}

Palavras-chave: Diabetes mellitus. Dieta com restrição de carboidratos. Dieta rica em proteínas e baixa em carboidratos. Dieta com restrição de gorduras. Dietoterapia.

\begin{abstract}
This study aimed to evaluate, through a literature review, evidence of the use of diets with carbohydrate restriction in the management of diabetes mellitus (DM). Electronic and manual searches were conducted until April 2019, including works published from 2008 onwards. Studies on in vitro and animal research, literature reviews, books, monographs, dissertations, theses, case studies and case reports were excluded. The remaining articles were submitted to analysis of their methodological quality by the five-point JADAD Scale. Nineteen randomized studies with an average quality of three points were selected and analyzed regarding the types of diet used, adherence, type of DM, time of intervention, reported consumption of carbohydrates and results observed for the control and intervention group. Among the parameters chosen to measure the possible effects of diets, weight and BMI changes, Hb1Ac, variability or glycemic control, lipid profile and changes in insulin doses or medication used stood
\end{abstract}


out. In four studies, no significant advantages were observed from a carbohydraterestricted diet, and 15 studies reported improvements in one or more parameters. Such results can represent a great advantage in adopting this dietary strategy in the management of DM and in preventing complications of the disease. However, there are limitations in the studies, which need to have their hypotheses verified in the long term, and additional research must be carried out to configure an official strategy in the control of DM.

Keywords: Diabetes mellitus. Carbohydrate-restricted diet. High-protein and low-carbohydrate diet. Fat-restriction diet. Diet therapy. 


\section{INTRODUÇÃO}

O diabetes mellitus (DM), em virtude de sua elevada prevalência e morbidade, figura como um grande problema de saúde pública, devido às complicações que pode gerar, além da mortalidade prematura. ${ }^{1}$ É de conhecimento geral que o atraso no desenvolvimento e na progressão de tais complicações só pode ser alcançado com um tratamento adequado no qual se consiga manter um bom controle glicêmico, ou seja, glicemia dentro da meta na maior parte do tempo. ${ }^{2}$ Para tanto, é fundamental o uso de uma estratégia alimentar saudável, mas também específica para tais pacientes, e a quantidade e a qualidade dos carboidratos da dieta há muito vêm sendo notadamente importante fator dietético envolvido no controle glicêmico. ${ }^{3}$

Apesar de todos os avanços no campo da Medicina e da Farmacologia, o manejo do DM ainda está longe de ser adequado. Atualmente, a média global de hemoglobina glicada (HbA1c) em pacientes diabéticos é de $8,2 \%$, sendo que a American Diabetes Association (ADA) estabelece que valores menores que 7\% são ideais para reduzir o risco de complicações. O maior desafio em atingir as metas esperadas para esse parâmetro de controle do DM é a dificuldade em controlar a glicemia pós-prandial, em virtude do descompasso entre a absorção de carboidratos e a ação da insulina, que tipicamente ocorre após as refeições nos portadores de diabetes tipo 1 (DM 1), bem como a quantidade e qualidade dos carboidratos consumidos nas refeições, no caso de indivíduos com diabetes do tipo 2 (DM 2). ${ }^{4,5}$ Ainda assim, a Sociedade Brasileira de Diabetes (SBD), em suas diretrizes 2017-2018, recomenda uma dieta baseada em 45 a 60\% constituída por carboidratos, e não menos que 130 gramas de carboidratos por dia, sendo que no máximo 5\% sejam provenientes da sacarose. ${ }^{6}$

O DM reflete uma perturbação no eixo glicose-insulina do metabolismo, com ausência da produção do hormônio em um tipo da doença (DM 1); no outro, a resistência à insulina é a característica definidora (DM 2). Em ambos os casos, o problema se encontra na passagem da glicose sanguínea para o interior das células. ${ }^{7}$ Por isso, espera-se que a restrição de carboidratos seja a principal estratégia de manejo do DM, sendo que esta foi a primeira abordagem a ser utilizada antes mesmo da descoberta da insulina. ${ }^{8}$ Do ponto de vista fisiológico, pode-se argumentar que os carboidratos consumidos na dieta devem ser controlados para alcançar um bom controle glicêmico em pacientes com DM.,10

Segundo a ADA, em um consenso publicado em 2019, reduzir a quantidade total de carboidratos para indivíduos com DM é a estratégia que apresentou maior quantidade de evidências para a melhora da glicemia, e podem ser aplicados diversos padrões alimentares, de acordo com a necessidade e preferência de cada indivíduo. ${ }^{11}$ Tal posicionamento se contrapõe às supracitadas recomendações da SBD. ${ }^{6}$

Sabendo que o DM pode trazer graves complicações à saúde e comprometer a qualidade de vida, e que ainda não há um controle satisfatório para a maioria dos portadores da doença, ${ }^{12,13}$ se faz necessário atualizar os profissionais da área da saúde, para que forneçam uma estratégia eficaz no controle dessa doença. Assim sendo, o objetivo deste estudo foi avaliar, por meio de uma revisão da literatura, evidências da utilização de dietas com restrição de carboidratos no manejo do DM.

\section{MÉTODOS}

A presente revisão de literatura foi elaborada segundo as recomendações PRISMA (Preferred Reporting Items for Systematic Reviews and Meta-Analyses). ${ }^{14}$ A pesquisa incluiu ensaios clínicos randomizados publicados a partir do ano de 2008 nos idiomas inglês, português e espanhol. Foram utilizadas as bases de dados LILACSBIREME (Base de Dados da Literatura Latino-Americana em Ciência da Saúde), SciELO (Scientific Eletronic Library Online) e PubMed (mantido pela National Library of Medicine). O período de busca foi entre agosto de 2018 e junho de 2019. 
A busca foi realizada por meio das palavras-chave baseadas nos Descritores em Ciências da Saúde (DeCS) nos idiomas português: diabetes mellitus, dieta com restrição de carboidratos, dieta rica em proteínas e baixa em carboidratos, dieta com restrição de gorduras, dietoterapia; e seus respectivos termos em inglês: diabetes mellitus; diet, carbohydrate restricted; diet, high protein low carbohydrate; diet, fat-restricted; diet therapy. Para a seleção dos artigos, realizou-se a leitura atenta do título e resumo dos mesmos, e se considerados relevantes para o estudo, procedia-se à leitura interpretativa completa do estudo. Foram acrescidos aos artigos escolhidos após esta etapa, outros trabalhos buscados manualmente, tanto em referências de estudos já selecionados, quanto em buscas assistemáticas do tema. Tal estratégia foi utilizada a fim de recuperar estudos originais/principais de cada intervenção.

Posteriormente, foram excluídos os estudos de pesquisas in vitro e em animais, bem como as revisões de literatura, estudos observacionais, estudos de caso e relatos de caso. Também foram excluídos artigos que não atingiram a pontuação de qualidade excelente na Escala JADAD cinco pontos (JADAD score on a 5-point scale). ${ }^{15}$ Trata-se de um mecanismo de avaliação da qualidade metodológica de trabalhos científicos, um dos principais recursos utilizados para avaliar a qualidade de ensaios clínicos randomizados, ${ }^{16}$ dividido em duas partes. Na primeira, acumula-se a pontuação máxima de três pontos, por meio de respostas opostas (sim - 1 ponto; não - 0 ponto) para os critérios: (1) randomização, (2) duplo-cego e (3) perdas (dropouts). Na segunda etapa, soma-se a pontuação, considerando dois critérios: (4) método de randomização adequado e (5) cegamento adequado. Quando um estudo pontua dois ou menos, é considerado de má qualidade; e quando soma acima de dois pontos, é avaliado com qualidade excelente. ${ }^{15}$

\section{RESULTADOS}

Na busca sistemática foram localizados 147 artigos, sendo 19 excluídos por serem resultados duplicados. Pela busca manual, foram encontrados e incluídos 16 artigos, resultando em 150 estudos, dos quais foram desconsiderados 131 pelos critérios de exclusão. No final do processo, restaram 19 artigos. Para a representação dos resultados, utilizou-se o fluxograma do método PRISMA, conforme figura 1.

Figura 1. Fluxograma do processo de seleção de artigos acerca da utilização de dietas com restrição de carboidratos no manejo do diabetes mellitus.

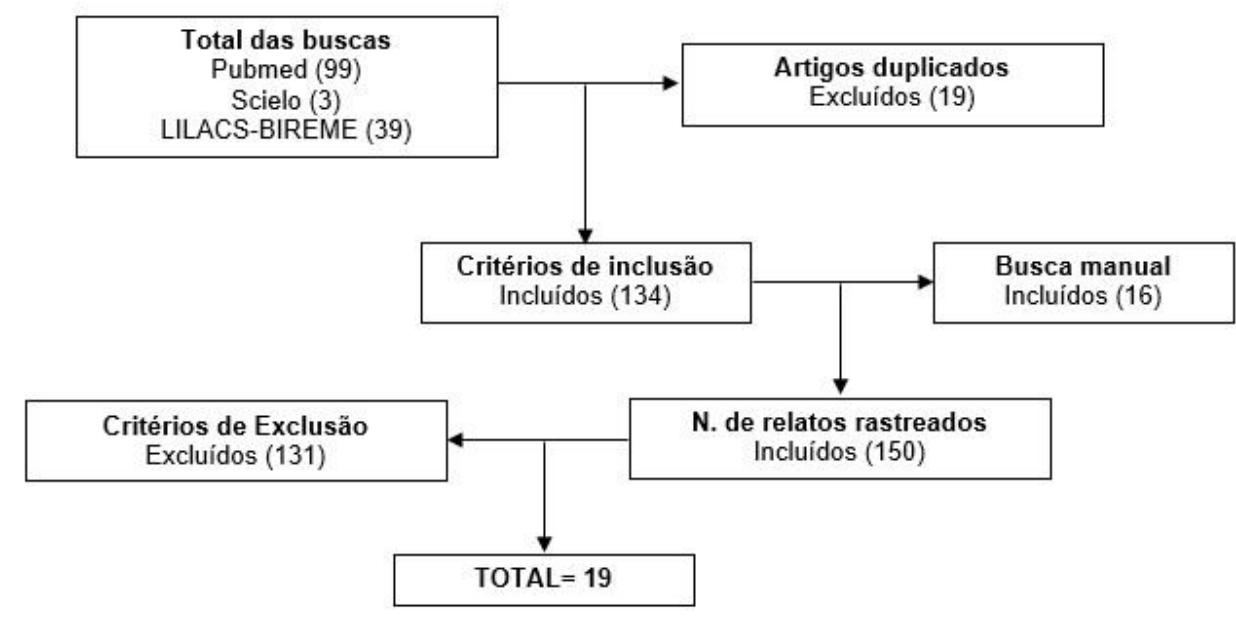

De acordo com a Escala JADAD, a média de qualidade dos estudos selecionados na presente revisão sistemática foi $3(\mathrm{DP}=0,58)$, significando que podem ser considerados de qualidade e que incluíram fatores importantes para a realização de um ensaio clínico randomizado. Dentre os itens apontados como 
fundamentais na qualidade dos estudos, ${ }^{15}$ todos os artigos fizeram uso do método de randomização, e a maioria $(n=15)$ descreveu o procedimento. As perdas foram apresentadas em todos os estudos, embora nenhum fosse duplo-cego.

As características dos estudos aqui incluídos estão descritas no quadro 1. Os estudos selecionados foram publicados entre os anos de 2008 e 2018. O tamanho amostral variou de 10 a 227 indivíduos adultos, portadores de DM do tipo 1 ou 2. O tempo de intervenção dos estudos variou entre 18 dias e dois anos de duração.

Todos os indivíduos dos grupos intervenção receberam dietas de baixo teor de carboidrato, sendo que a quantidade total desse macronutriente variou entre 14 e 49\% do valor energético total (VET) da dieta, ou entre 20 e $130 \mathrm{~g}$ de carboidratos por dia. Nas dietas aplicadas aos grupos controle em cada estudo, a quantidade de carboidrato variou entre 40 a 65\% do VET da dieta em alguns estudos; em outros, foram classificadas como compostas de uma quantidade igual ou superior a 130g, 165g ou $250 \mathrm{~g}$ de carboidratos por dia. As dietas utilizadas para os grupos intervenção e controle foram classificadas em termos de valor energético como sendo isocalóricas, não isocalóricas ou de déficit calórico similar para cada participante, de acordo com o gasto energético total individual.

Quadro 1. Principais características dos estudos selecionados sobre dietas com restrição de carboidratos no manejo do diabetes mellitus.

\begin{tabular}{|c|c|c|c|c|c|c|c|c|}
\hline Fonte & Tipo de Estudo & $\begin{array}{l}\text { Tipos de } \\
\text { Dieta }\end{array}$ & $\mathrm{Ne} \%$ de adesão & $\begin{array}{l}\text { Tipo de } \\
\text { Diabetes }\end{array}$ & \begin{tabular}{|c|} 
Tempo de \\
intervenção
\end{tabular} & $\begin{array}{l}\text { VET das } \\
\text { dietas }\end{array}$ & $\begin{array}{c}\text { Composição das } \\
\text { dietas }\end{array}$ & Resultados observados \\
\hline $\begin{array}{l}\text { Wang } \\
\text { et al. } \\
(2018)^{17}\end{array}$ & $\begin{array}{l}\text { Ensaio clínico, } \\
\text { prospectivo, } \\
\text { randomizado, } \\
\text { cego, controlado }\end{array}$ & $\begin{array}{l}\text { Gl: LCHF } \\
\text { GC: LFD }\end{array}$ & $\begin{array}{c}\text { I: Gl}=28 \text { e GC=28 } \\
\begin{array}{c}F: G \mid=24(85,7 \%) \text { e } \\
G C=25 \\
(89,3 \%)\end{array}\end{array}$ & $\mathrm{DM} 2$ & 3 meses & \begin{tabular}{|c} 
Dietas \\
isocalóricas
\end{tabular} & $\begin{array}{c}\text { Gl: } 39 \% \text { CHO e } \\
42 \% \text { LIP } \\
\text { GC: } 56 \% \text { CHO e } \\
\text { 26\% LIP }\end{array}$ & $\begin{array}{l}\text { LCHF se mostrou superior em } \\
\text { relação à LFD no controle } \\
\text { glicêmico. Regula índices de } \\
\text { colesterol, reduziu IMC, diminuiu } \\
\text { dose de insulina }(p<0,05) \text {. }\end{array}$ \\
\hline $\begin{array}{l}\text { Watson } \\
\text { et al. } \\
(2018)^{18}\end{array}$ & $\begin{array}{l}\text { Ensaio clínico } \\
\text { randomizado }\end{array}$ & $\begin{array}{l}\text { Gl: LCHP } \\
\text { GC: HC }\end{array}$ & $\begin{array}{c}\text { I: Gl}=32 \text { e } G C=31 \\
F: G l=23(71,9 \%) \text { e } \\
G C=21(67,4 \%)\end{array}$ & $\mathrm{DM} 2$ & 24 semanas & \begin{tabular}{|l} 
Dietas \\
isocalóricas
\end{tabular} & $\begin{array}{c}\text { Gl: } 34 \% \text { CHO, } \\
\text { 29\% PTN, } 31 \% \\
\text { LIP } \\
\text { GC: } 48 \% \text { CHO, } \\
\text { 21\% PTN, 24\% } \\
\text { LIP }\end{array}$ & $\begin{array}{l}\text { Redução de peso, melhora na } \\
\text { Hb1Ac, bem-estar psicológico } \\
\text { tanto na dieta LCHP quanto na } \\
\text { HC. Dieta LCHP demonstrou } \\
\text { melhorar vitalidade }(p<0,05) \text {. }\end{array}$ \\
\hline $\begin{array}{l}\text { Tay et al. } \\
(2017)^{19}\end{array}$ & $\begin{array}{l}\text { Ensaio clínico } \\
\text { randomizado, } \\
\text { cego, controlado }\end{array}$ & $\begin{array}{l}\text { Gl: LCD } \\
\text { GC: LFD }\end{array}$ & $\begin{array}{c}I: G \mid=58 \\
G C=57 \\
F: G \mid=33(56,9 \%) \\
G C=28(49,1 \%)\end{array}$ & DM 2 & 2 anos & \begin{tabular}{|} 
Dietas \\
isocalóricas
\end{tabular} & $\begin{array}{l}\text { GI: } 14 \% \text { CHO, } \\
28 \% \text { PTN e } 58 \% \\
\text { LIP (<10\% sat.) } \\
\text { GC: } 53 \% \text { CHO, } \\
17 \% \text { PTN, } 30 \% \\
\text { LIP (<10\% sat.) }\end{array}$ & $\begin{array}{l}\text { Ambas as dietas alcançaram } \\
\text { perda de peso e reduções de } \\
\text { HbA1c. A LCD sustentou } \\
\text { maiores reduções nos usos de } \\
\text { medicação para diabetes, e em } \\
\text { melhorias na estabilidade de } \\
\text { glicemia e perfil lipídico do } \\
\text { sangue, sem danos renais, } \\
\text { sugerindo otimização do } \\
\text { gerenciamento de DM } 2 \\
(p<0,05) \text {. }\end{array}$ \\
\hline $\begin{array}{l}\text { Ranjan } \\
\text { et al. } \\
(2017)^{20}\end{array}$ & $\begin{array}{l}\text { Ensaio clínico, } \\
\text { cruzado, } \\
\text { randomizado e } \\
\text { aberto }\end{array}$ & $\begin{array}{l}\text { Gl: LCD } \\
\text { GC: HCD }\end{array}$ & $\begin{array}{c}|: G|=5 \\
G C=5 \\
F: G \mid=5(100 \%) \\
G C=5(100 \%)\end{array}$ & DM 1 & 18 dias & \begin{tabular}{|l} 
Dietas \\
isocalóricas
\end{tabular} & $\begin{array}{l}\text { Gl: } \leq 50 \mathrm{~g} \\
\mathrm{CHO} / \mathrm{dia} \\
\mathrm{GC}: \geq 250 \mathrm{~g} \\
\mathrm{CHO} / \mathrm{dia}\end{array}$ & $\begin{array}{c}\text { O LCD resultou em mais tempo } \\
\text { de euglicemia, menos tempo de } \\
\text { hipoglicemia e menor } \\
\text { variabilidade de glicose que } \\
\text { HCD sem alterar as } \\
\text { concentrações de glicose ( } p< \\
0,05) \text {. } \\
\end{array}$ \\
\hline $\begin{array}{l}\text { Saslow } \\
\text { et al. } \\
(2017)^{21}\end{array}$ & $\begin{array}{c}\text { Ensaio clínico, } \\
\text { prospectivo, } \\
\text { randomizado }\end{array}$ & $\begin{array}{l}\text { Gl: } \\
\text { VLCKD } \\
\text { GC: } \\
\text { MCCR }\end{array}$ & $\begin{array}{l}\mathrm{I}: \mathrm{Gl}=16 \mathrm{GC}=18 \\
\mathrm{~F}: \mathrm{Gl}=14(87,5 \%) \\
\mathrm{GC}=15(83,3 \%)\end{array}$ & $\mathrm{DM} 2$ & 12 meses & \begin{tabular}{|l} 
Dietas não \\
isocalóricas
\end{tabular} & $\begin{array}{c}\text { Gl: 20-50 g de net } \\
\text { CHO } \\
\text { GC: } 165 \text { g de } \\
\text { CHO (40 - 50\% } \\
\text { VET), hipocalórica } \\
\text { (-500 Kcal), LFD } \\
\end{array}$ & $\begin{array}{l}\text { No grupo VLCKD houve maiores } \\
\text { reduções na HbA1c, maior } \\
\text { perda de peso e redução de } \\
\text { medicamentos do que aqueles } \\
\text { instruídos a seguir uma dieta } \\
\text { MCCR. }\end{array}$ \\
\hline
\end{tabular}


Quadro 1. Principais características dos estudos selecionados sobre dietas com restrição de carboidratos no manejo do diabetes mellitus. (Cont).

\begin{tabular}{|c|c|c|c|c|c|c|c|c|}
\hline Fonte & Tipo de Estudo & $\begin{array}{c}\text { Tipos de } \\
\text { Dieta }\end{array}$ & N e \% de adesão & $\begin{array}{l}\text { Tipo de } \\
\text { Diabetes }\end{array}$ & $\begin{array}{l}\text { Tempo de } \\
\text { intervenção }\end{array}$ & $\begin{array}{l}\text { VET das } \\
\text { dietas }\end{array}$ & $\begin{array}{c}\text { Composição das } \\
\text { dietas }\end{array}$ & Resultados observados \\
\hline $\begin{array}{l}\text { Wycherle } \\
\text { y et al. } \\
(2016)^{22}\end{array}$ & $\begin{array}{l}\text { Ensaio clínico, } \\
\text { prospectivo, } \\
\text { randomizado }\end{array}$ & $\begin{array}{l}\text { Gl: } \\
\text { VLCKD } \\
\text { GC: HCD }\end{array}$ & $\begin{array}{l}\text { I: GI=58 GC=57 } \\
F: G \mid=41(70,7 \%) \\
G C=37(64,9 \%)\end{array}$ & DM 2 & 12 meses & $\begin{array}{l}\text { Dietas } \\
\text { isocalóricas }\end{array}$ & $\begin{array}{l}\text { GI: 14\% CHO, } \\
28 \% \text { PTN, 58\% } \\
\text { LIP (<10\% sat) } \\
\text { GC: } 53 \% \text { CHO, } \\
17 \% \text { PTN, 30\% } \\
\text { LIP (<10\% sat) }\end{array}$ & $\begin{array}{l}\text { Ambas dietas, combinadas com } \\
\text { melhora geral nos hábitos de } \\
\text { vida, apresentaram benefícios } \\
\text { similares na redução de peso, } \\
\text { melhora da Hb1Ac e função } \\
\text { endotelial }(p<0,05) \text {. }\end{array}$ \\
\hline $\begin{array}{l}\text { Raygan } \\
\text { et al. } \\
(2016)^{23}\end{array}$ & $\begin{array}{l}\text { Ensaio clínico, } \\
\text { prospectivo, } \\
\text { randomizado }\end{array}$ & $\begin{array}{l}\text { Gl: LCD } \\
\text { GC: HCD }\end{array}$ & $\begin{array}{l}\text { I: } G \mid=28 \text { GC=28 } \\
F: G \mid=28(100 \%) \\
G C=28(100 \%)\end{array}$ & DM 2 & 8 semanas & $\begin{array}{l}\text { Déficit } \\
\text { calórico } \\
\text { similar }\end{array}$ & $\begin{array}{c}\text { Gl: } 43-49 \% \text { CHO, } \\
\text { 36-40\% LIP e 14- } \\
\text { 17\% PTN } \\
\text { GC: } 60-65 \% \text { CHO, } \\
\text { 20-25\% LIP e 14- } \\
\text { 17\% PTN }\end{array}$ & $\begin{array}{l}\text { A LCD apresentou melhores } \\
\text { resultados na redução da } \\
\text { glicemia de jejum, e maior } \\
\text { sensibilidade à proteína C- } \\
\text { reativa ( } p<0,05 \text { ). A HCD } \\
\text { apresentou maiores } \\
\text { concentrações de glutationa e } \\
\text { capacidade antioxidante } \\
\text { (p<0,05). Não houve diferença } \\
\text { significativa entre outros } \\
\text { marcadores metabólicos. }\end{array}$ \\
\hline $\begin{array}{c}\text { Sato } \\
\text { et al. } \\
(2016)^{24}\end{array}$ & $\begin{array}{l}\text { Ensaio clínico, } \\
\text { prospectivo, } \\
\text { randomizado }\end{array}$ & $\begin{array}{l}\text { Gl: LCD } \\
\text { GC: Dieta } \\
\text { hipocalór } \\
\quad \text { ica }\end{array}$ & $\begin{array}{l}\mathrm{I}: \mathrm{Gl}=33 \mathrm{GC}=33 \\
\mathrm{~F}: \mathrm{Gl}=30(90,9 \%) \\
\mathrm{GC}=32(97 \%)\end{array}$ & DM 2 & 6 meses & $\begin{array}{l}\text { Dietas não } \\
\text { isocalóricas }\end{array}$ & $\begin{array}{c}\text { Gl: <130 g de } \\
\text { CHO /dia } \\
\text { GC: Dieta } \\
\text { hipocalórica ( } 28 \\
\text { kcal x peso ideal) }\end{array}$ & $\begin{array}{c}\text { Houve maior redução na } \\
\text { concentração de HbA1c no } \\
\text { grupo LCD }(p<0,05) \text {, como } \\
\text { também maior redução no IMC } \\
(p<0,05) \text {. }\end{array}$ \\
\hline $\begin{array}{l}\text { Krebs } \\
\text { et al. } \\
(2016)^{25}\end{array}$ & $\begin{array}{l}\text { Ensaio clínico, } \\
\text { prospectivo, } \\
\text { randomizado }\end{array}$ & $\begin{array}{l}\text { Gl: LCD } \\
\text { GC: dieta } \\
\text { livre com } \\
\text { contage } \\
\text { m de } \\
\text { carboidr } \\
\text { atos }\end{array}$ & $\begin{array}{c}|: G|=5 \\
G C=5 \\
F: G I=5(100 \%) \\
G C=5(100 \%)\end{array}$ & $\begin{array}{c}\text { DM } 1 \\
\text { (adultos) }\end{array}$ & $\begin{array}{c}12 \\
\text { semanas }\end{array}$ & $\begin{array}{l}\text { Dietas não } \\
\text { isocalóricas }\end{array}$ & $\begin{array}{c}\text { Gl: } \mathrm{CHO}<75 \\
\text { g/dia }\end{array}$ & $\begin{array}{l}\text { Diminuição nas concentrações } \\
\text { de Hb1Ac sem efeitos adversos } \\
\text { no grupo intervenção }(p<0,05) \text {. }\end{array}$ \\
\hline $\begin{array}{l}\text { Brinkwor } \\
\text { th et al. } \\
(2016)^{26}\end{array}$ & $\begin{array}{l}\text { Ensaio clínico, } \\
\text { prospectivo, } \\
\text { randomizado }\end{array}$ & $\begin{array}{l}\text { Gl: LCD } \\
\text { GC: HCD }\end{array}$ & $\begin{array}{c}|: G|=58 \text { e } \\
G C=57 \\
F: G \mid=41 \\
(70,7 \% \%) \\
G C=37(64,9 \%)\end{array}$ & DM 2 & 1 ano & $\begin{array}{c}\text { Dietas } \\
\text { isocalóricas }\end{array}$ & $\begin{array}{c}\text { Gl: } 14 \% \text { CHO } \\
\text { (<50 g/dia), 28\% } \\
\text { PTN e 58\% LIP } \\
\text { (<10\% sat) } \\
\text { GC: } 53 \% \text { CHO, } \\
\text { 17\% PTN e <30\% } \\
\text { de LIP (<10\% sat) }\end{array}$ & $\begin{array}{c}\text { Não houve diferença } \\
\text { significativa de resultados perda } \\
\text { de peso, qualidade de vida e } \\
\text { estado psicológico. Ambas as } \\
\text { dietas, dentro de um programa } \\
\text { de modificação de estilo de vida } \\
\text { que inclui treinamento físico, } \\
\text { melhoram esses parâmetros. }\end{array}$ \\
\hline $\begin{array}{l}\text { Tay et al. } \\
(2014)^{27}\end{array}$ & $\begin{array}{l}\text { Ensaio clínico, } \\
\text { prospectivo, } \\
\text { randomizado }\end{array}$ & $\begin{array}{l}\text { Gl: LCD } \\
\text { GC: HCD }\end{array}$ & $\begin{array}{l}|: G|=58 G C=57 \\
F: G \mid=46(79,3 \%) \\
G C=47(82,4 \%)\end{array}$ & DM2 & $\begin{array}{c}24 \\
\text { semanas }\end{array}$ & $\begin{array}{c}\text { Dietas } \\
\text { isocalóricas }\end{array}$ & $\begin{array}{c}\text { Gl: } 14 \% \text { CHO } \\
\text { [<50 g/dia], 28\% } \\
\text { PTN, e } 58 \% \text { LIP } \\
\text { (<10\% sat) } \\
\text { GC: } 53 \% \text { CHO, } \\
17 \% \text { PTN, e } 30 \% \\
\text { LIP }\end{array}$ & $\begin{array}{c}\text { Ambos os grupos melhoraram } \\
\text { as concentrações de LDL, PA e } \\
\text { glicemia de jejum }(p \geq 0,1) \text {. No } \\
\text { grupo LCD houve melhora nas } \\
\text { concentrações de Hb1Ac e } \\
\text { variabilidade glicêmica, bem } \\
\text { como aumento nas } \\
\text { concentrações HDL ( } p<0,05) \text {. }\end{array}$ \\
\hline $\begin{array}{l}\text { Rock et } \\
\text { al. } \\
(2014)^{28}\end{array}$ & $\begin{array}{l}\text { Ensaio clínico } \\
\text { prospectivo, } \\
\text { controlado } \\
\text { randomizado }\end{array}$ & $\begin{array}{l}\text { GI1: } \\
\text { LCHF } \\
\text { GI2: LFD } \\
\text { GC: Dieta } \\
\text { do } \\
\text { program } \\
\text { a UC }\end{array}$ & $\begin{array}{c}\text { I: GI1 }=74, G \mid 2=77, \\
\text { GC }=76 \\
F: G \mid 1=73(98,6 \%), \\
G \mid 2=76(98,7 \%), \\
G C=76(100 \%)\end{array}$ & DM 2 & 1 ano & $\begin{array}{l}\text { Déficit } \\
\text { calórico } \\
\text { similar }\end{array}$ & $\begin{array}{c}\text { GI1: } 45 \% \text { CHO, } \\
\text { 25\% PTN e 30\% } \\
\text { LIP } \\
\text { GI2: } 60 \% \text { CHO, } \\
\text { 20\% PTN e 20\% } \\
\text { LIP } \\
\text { GC: } 55 \% \text { CHO, } \\
\text { 15\% PTN e } 30 \% \\
\text { LIP } \\
\end{array}$ & $\begin{array}{l}\text { Os programas LCHF e LFD } \\
\text { apresentaram melhores } \\
\text { resultados no controle glicêmico } \\
\text { e perda de peso do que o } \\
\text { Programa UC proposto pela } \\
\text { Universidade. }\end{array}$ \\
\hline $\begin{array}{c}\text { Jonasson } \\
\text { et al. } \\
(2014)^{29}\end{array}$ & $\begin{array}{l}\text { Ensaio clínico } \\
\text { prospectivo, } \\
\text { controlado } \\
\text { randomizado }\end{array}$ & $\begin{array}{l}\text { GI: LCD } \\
\text { GC: LFD }\end{array}$ & $\begin{array}{l}|: G|=30 \mathrm{GC}=31 \\
F: G \mid=30(100 \%) \\
G C=31(100 \%)\end{array}$ & DM 2 & 2 anos & $\begin{array}{c}\text { Dietas } \\
\text { isocalóricas }\end{array}$ & $\begin{array}{c}\text { Gl: } 25 \% \mathrm{CHO} e \\
49 \% \text { LIP } \\
\text { GC: } 49 \% \text { CHO } \\
\text { 29\% LIP }\end{array}$ & $\begin{array}{l}\text { Apenas LCD foi encontrado } \\
\text { significativamente para } \\
\text { melhorar estado inflamatório } \\
\text { subclínico no diabetes tipo } 2 \\
\qquad(p<0,05) \text {. }\end{array}$ \\
\hline
\end{tabular}


Quadro 1. Principais características dos estudos selecionados sobre dietas com restrição de carboidratos no manejo do diabetes mellitus. (Cont).

\begin{tabular}{|c|c|c|c|c|c|c|c|c|}
\hline Fonte & Tipo de Estudo & $\begin{array}{l}\text { Tipos de } \\
\text { Dieta }\end{array}$ & N e \% de adesão & $\begin{array}{l}\text { Tipo de } \\
\text { Diabetes }\end{array}$ & \begin{tabular}{|c|} 
Tempo de \\
intervenção
\end{tabular} & $\begin{array}{l}\text { VET das } \\
\text { dietas }\end{array}$ & $\begin{array}{c}\text { Composição das } \\
\text { dietas }\end{array}$ & Resultados observados \\
\hline $\begin{array}{l}\text { Guldbran } \\
\text { d et al. } \\
(2014)^{30}\end{array}$ & $\begin{array}{l}\text { Ensaio clínico, } \\
\text { prospectivo, } \\
\text { randomizado }\end{array}$ & $\begin{array}{l}\mathrm{Gl}=\mathrm{LCD} \\
\mathrm{GC}=\mathrm{LFD}\end{array}$ & $\begin{array}{l}\mathrm{I}: \mathrm{Gl}=30 \mathrm{GC}=30 \\
\mathrm{~F}: \mathrm{Gl}=25(83,3 \%) \\
\mathrm{GC}=29(96,7 \%)\end{array}$ & $\mathrm{DM} 2$ & 2 anos & \begin{tabular}{|c} 
Dietas \\
isocalóricas
\end{tabular} & $\begin{array}{c}\text { GI: 20\% CHO, } \\
\text { 30\% PTN e 50\% } \\
\text { LIP } \\
\text { GC: } 55-60 \% \text { CHO, } \\
\text { 10-15\% PTN e } \\
\text { 30\% LIP } \\
\end{array}$ & $\begin{array}{l}\text { Não houve diferenças } \\
\text { significativas entre perda de } \\
\text { peso nos dois grupos, mas após } \\
\text { um ano de intervenção, houve } \\
\text { melhora na qualidade de vida } \\
\text { do grupo LCD. }\end{array}$ \\
\hline $\begin{array}{c}\text { Saslow et } \\
\text { al. } \\
(2014)^{31}\end{array}$ & $\begin{array}{l}\text { Ensaio clínico, } \\
\text { prospectivo, } \\
\text { randomizado }\end{array}$ & $\begin{array}{l}\text { Gl: } \\
\text { VLCKD } \\
\text { GC: } \\
\text { MCCR }\end{array}$ & $\begin{array}{l}\mathrm{I}: \mathrm{Gl}=16 \mathrm{GC}=18 \\
\mathrm{~F}: \mathrm{Gl}=15(93,8 \%) \\
\mathrm{GC}=18(100 \%)\end{array}$ & $\mathrm{DM} 2$ & 3 meses & $\begin{array}{l}\text { Dietas não } \\
\text { isocalóricas }\end{array}$ & $\begin{array}{c}\text { Gl: } 20-50 \text { g de } \\
\text { netCHO } \\
\text { GC: } 165 \text { g de } \\
\text { CHO (40 - 50\% } \\
\text { VET), hipocalórica } \\
\text { (-500 Kcal), LFD }\end{array}$ & $\begin{array}{l}\text { Diminuição nas concentrações } \\
\text { de HbA1c e nas doses de } \\
\text { medicações }(p<0,05) \text { e maior } \\
\text { perda de peso }(p=0,09) \text { no } \\
\text { grupo VLCKD. }\end{array}$ \\
\hline $\begin{array}{l}\text { Goldstei } \\
\text { n et al. } \\
(2011)^{32}\end{array}$ & $\begin{array}{l}\text { Ensaio clínico, } \\
\text { randomizado, } \\
\text { controlado }\end{array}$ & $\begin{array}{l}\text { Gl: ATK } \\
(\text { LCD) } \\
\text { GC: Dieta } \\
\text { recomen } \\
\text { dada } \\
\text { pela ADA } \\
\end{array}$ & $\begin{array}{l}\text { I: Gl=26 GC=26 } \\
F: G \mid=14(53,8 \%) \\
G C=16(61,5 \%)\end{array}$ & $\mathrm{DM} 2$ & 1 ano & $\begin{array}{l}\text { Dietas não } \\
\text { isocalóricas }\end{array}$ & $\begin{array}{c}\text { Gl: } 25 \text { g de } \\
\text { CHO/40 g de } \\
\text { CHO } \\
\text { GC: } 50-60 \% \text { CHO, } \\
\text { 10\%-20\% PTN e } \\
\text { 30\% LIP } \\
\end{array}$ & $\begin{array}{l}\text { Não houve vantagem } \\
\text { estatisticamente significativa em } \\
\text { termos de perda de peso ou } \\
\text { controle glicêmico para a dieta } \\
\text { tipo Atkins } \\
(p<0,05) .\end{array}$ \\
\hline $\begin{array}{l}\text { Larsen et } \\
\text { al. } \\
(2011)^{33}\end{array}$ & $\begin{array}{l}\text { Estudo } \\
\text { prospectivo, } \\
\text { controlado, } \\
\text { randomizado }\end{array}$ & $\begin{array}{l}\text { Gl: LCHP } \\
\text { GC: HCD }\end{array}$ & $\begin{array}{c}\mathrm{I}: \mathrm{Gl}=53 \\
\mathrm{GC}=46 \\
\mathrm{~F}: \mathrm{Gl}=48(90,6 \%) \\
\mathrm{GC}=45(97,8 \%)\end{array}$ & $\mathrm{DM} 2$ & 1 ano & \begin{tabular}{|l} 
Dietas \\
isocalóricas
\end{tabular} & $\begin{array}{c}\text { Gl: } 40 \% \text { CHO, } \\
30 \% \text { PTN e } 30 \% \\
\text { LIP } \\
\text { GC: } 55 \% \text { CHO, } \\
\text { 15\% PTN e 30\% } \\
\text { LIP }\end{array}$ & $\begin{array}{c}\text { Estes resultados sugerem que } \\
\text { não existe nenhum benefício } \\
\text { metabólico superior, a longo } \\
\text { prazo, de uma dieta rica em } \\
\text { proteínas em relação a uma } \\
\text { dieta com alto teor de } \\
\text { carboidratos no tratamento do } \\
\text { diabetes tipo } 2(p=0,44) \text {. }\end{array}$ \\
\hline $\begin{array}{c}\text { Davis et } \\
\text { al. } \\
(2009)^{34}\end{array}$ & $\begin{array}{l}\text { Ensaio clínico } \\
\text { randomizado }\end{array}$ & $\begin{array}{l}\text { GI: LCD } \\
\text { GC: LFD }\end{array}$ & $\begin{array}{c}\mathrm{I}: \mathrm{Gl}=55 \\
\mathrm{GC}=50 \\
\mathrm{~F}: \mathrm{Gl}=47(85,4 \%) \\
\mathrm{GC}=44(88 \%)\end{array}$ & $\mathrm{DM} 2$ & 1 ano & $\begin{array}{l}\text { Dietas não } \\
\text { isocalóricas }\end{array}$ & $\begin{array}{l}\text { Gl: } 20-25 \text { g de } \\
\text { CHO/dia com } \\
\text { incremento de } 5 g \\
\text { por semana } \\
\text { GC: } 25 \% \text { do VET } \\
\text { em LIP }\end{array}$ & $\begin{array}{l}\text { A perda de peso ocorreu mais } \\
\text { rapidamente no grupo } L C \text { do } \\
\text { que no grupo } L F(p<0,05) \text {, mas } \\
\text { em um ano uma redução de } \\
\text { peso similar de foi observada } \\
\text { em ambos os grupos dietéticos. } \\
\text { Não houve mudança } \\
\text { significativa em } A 1 C \text { em } \\
\text { qualquer grupo em um ano. } \\
\text { Não houve alteração na pressão } \\
\text { arterial, mas um maior aumento } \\
\text { no HDL foi observado no grupo } \\
\text { LCD }(p<0,05) \text {. }\end{array}$ \\
\hline
\end{tabular}

Legenda: Gl: grupo intervenção; GC: grupo controle; I: início do estudo; F: fim do estudo; Dietas: LCHF: low carb high fat; LFD: low fat diet; LCHP: Low carb high protein; HC: High carb; LCD: low carb diet; HCD: high carb diet; VLCKD: Very low carb keto diet; MCCR: medium carbohydrate, low fat, calorie-restricted, carbohydrate counting diet; ATK: dieta Atkins; HCHGI: high carb high glicemic index; HCLGI: High carb low glicemic index; VET: Valor energético total; CHO: carboidratos; PTN: Proteínas; LIP: lipídios; sat: gorduras saturadas; net CHO: carboidratos líquidos.

A maioria dos estudos selecionados para análise na presente revisão abordou estratégias de intervenção com dietas com restrição de carboidratos em pacientes portadores de DM 2, e apenas dois artigos dentre os 19 selecionados direcionaram-se a pacientes com DM 1. Houve ainda grande variação no tempo de intervenção, sendo o menor tempo 18 dias e o maior, dois anos. Sobressaíram-se os estudos nos quais as dietas utilizadas para os grupos intervenção e controle foram isocalóricas, mas em alguns trabalhos as dietas tiveram um VET diferente ou relatou-se um déficit calórico similar sob o gasto energético total de cada indivíduo. Além disso, houve grande variabilidade entre as proporções dos macronutrientes que compuseram cada dieta aplicada, bem como na nomenclatura adotada para classificar cada dieta. 


\section{DISCUSSÃO}

A partir dos resultados encontrados no presente estudo, observa-se uma escassez de artigos nos quais o impacto de dietas com baixo teor de carboidratos é averiguado em indivíduos com DM 1 em relação aos conduzidos com pacientes com DM 2. Corroborando tal achado, um trabalho de revisão de literatura publicado no ano de 2018 também relatou apenas um estudo randomizado realizado com indivíduos portadores de DM 1, publicado após o ano de 2009. ${ }^{36}$

Uma possível justificativa para esse resultado é o fato de que portadores de DM 1 podem monitorar a ingestão de carboidratos por meio da contagem de carboidratos, a fim de calcular as doses de insulina prérefeição, e assim controlar as concentrações de glicose pós-prandiais. Desta forma, os pacientes podem ser ensinados a avaliar o teor de carboidratos da refeição em gramas, e o quanto o teor a ser ingerido representará em dose de insulina a ser administrada para manter a normoglicemia. ${ }^{37} \mathrm{~A}$ partir disso, infere-se que saber fazer a contagem de carboidratos de forma correta e manter o monitoramento contínuo da glicemia possibilita ao indivíduo DM 1 obter ótimo controle glicêmico, sem a necessidade de restringir a quantidade de carboidratos da dieta. ${ }^{38}$

Contudo, alguns problemas têm sido observados na prática, sobretudo no que diz respeito a crianças com DM 1, que dependem de pais ou cuidadores. Há grande tendência em se subestimar a dose de insulina a ser administrada pela contagem de carboidratos, pelo temor de uma situação de hipoglicemia, principalmente em refeições maiores, o que tem resultado em concentrações de Hb1Ac e glicemia pósprandial elevadas. ${ }^{37,39,40}$ Além disso, resultados de estudos apontam uma inabilidade geral na precisão de contagem de carboidratos entre os indivíduos portadores de DM 1.37,41

Em relação ao tempo de intervenção de cada dieta, houve grande variação entre os artigos selecionados (entre 18 dias e 2 anos). A fim de reunir maior número de estudos acerca do tema abordado, um tempo mínimo de intervenção não foi estabelecido como critério de inclusão. Contudo, em outro estudo de revisão sistemática, ${ }^{42}$ os autores justificam a exclusão de estudos com tempo de intervenção inferior a 12 semanas pelo fato de que o principal objetivo do controle metabólico dos indivíduos com DM é reduzir as implicações dessa patologia em longo prazo. Esse tempo não se aplica para dois dentre os 19 artigos selecionados por este trabalho.

É importante ressaltar que 12 semanas é o tempo médio para que mudanças no tratamento do DM surtam efeitos no resultado da Hb1Ac, melhor parâmetro preditor de complicações no DM e fundamental na avaliação do controle glicêmico do paciente. ${ }^{43}$ Assim, estudos com um tempo de intervenção menor que este podem não ter efeitos tão significativos para que se afirme a qualidade da intervenção.

Dentre os artigos selecionados pelo presente trabalho cujo tempo de estudo foi inferior a 12 semanas, foram avaliados apenas parâmetros que sofrem variação em curto prazo, como glicemia de jejum, tempo de euglicemia, variabilidade glicêmica, sensibilidade à proteína C-reativa.

Em estudos com baixo tempo de intervenção, existe uma limitação em avaliar a adesão do indivíduo a um tipo de dieta, e se a mesma seria aplicável no longo prazo, bem como os possíveis efeitos que causaria. A adesão entre os indivíduos alocados nos grupos intervenção nos estudos selecionados variou entre 53,8\% e 100\%; e a adesão entre os indivíduos randomizados para o grupo controle variou entre 49,1\% e 100\%.

Uma questão importante a ser levantada a respeito da adesão à dieta é o quão desafiadora pode ser a adoção de uma dieta restritiva em quantidade de carboidratos para alguns indivíduos, principalmente quando mudanças menos drásticas na dieta e estilo de vida são recomendadas pelas atuais diretrizes brasileiras, mais fáceis de serem acompanhadas no longo prazo. ${ }^{44}$ Dentre os estudos selecionados, três relatam 100\% de 
adesão dos indivíduos tanto nos grupos controle quanto intervenção, sendo que estes utilizaram os menores tempos de intervenção (18 dias, 8 semanas e 12 semanas).

Noaks \& Windt relatam, em sua revisão de literatura, ${ }^{45}$ estudos que demonstram similaridade de adesão entre dietas low carb high fat (LCHF) e low fat high carb (LFHC), ${ }^{46-48}$ e um estudo que demonstra maior continuidade de seguimento em indivíduos que adotam a dieta LCHF em comparação àqueles que adotaram uma dieta hipocalórica e low fat..$^{49}$ Os autores concluem, portanto, que as evidências apontam que os pacientes parecem não achar mais difícil aderir a uma dieta LCHF do que a qualquer outra estratégia dietética. Acrescentam ainda, que devido à sua capacidade única de reduzir a fome, alguns pacientes podem achar que as dietas LCHF são mais facilmente sustentáveis do que as dietas de LFHC que requerem restrição calórica consciente.

As dietas aplicadas aos grupos intervenção e controle foram isocalóricas em dez dentre os 19 artigos selecionados; seis utilizaram dietas não isocalóricas e em três estudos foram calculadas individualmente as necessidades energéticas de cada indivíduo, e aplicado às mesmas um déficit calórico similar. Os autores que adotaram dietas isocalóricas no intuito de compará-las, ou aqueles que utilizaram um mesmo déficit calórico com base nas necessidades energéticas individuais, puderam observar de forma mais fidedigna os possíveis efeitos de se alterar as proporções de macronutrientes na dieta, visto que o balanço energético era similar entre os grupos. Em estudos nos quais as dietas não foram isocalóricas, não se pode isolar a possibilidade de que um maior déficit calórico tenha ocorrido para o grupo intervenção ou controle, e em virtude disso os resultados tenham favorecido uma ou outra dieta em termos de eficácia.

Dentre os artigos selecionados para a presente revisão, houve grande variabilidade no que se refere a definição e composição de cada dieta em termos de proporção de macronutrientes. As dietas com menor teor de carboidrato, entre 20 e 50g diárias ou representando até 14\% do VET diário, foram classificadas pelos autores como sendo very low carb keto diet (VLCKD), ou dieta cetogênica. Houve ainda estudos que, em suas dietas intervenção, compensaram a redução do teor de carboidratos elevando o teor de apenas um macronutriente, sendo proteína (low carb high protein, LCHP) ou lipídios (low carb high fat, LCHF), ou apenas incrementaram ambos em igual proporção (low carb diet, LCD). A maior parte das dietas do grupo controle foi nomeada pelos autores como sendo de alto teor de carboidratos (high carb diet, HCD) ou baixo teor de gorduras (low fat diet, LFD).

É possível encontrar na literatura diversas definições para uma dieta restrita em carboidratos, e a falta de consenso e padronização entre essas definições dificulta a comparação entre as dietas e seus benefícios. E embora haja variação entre estudos e autoridades, no consenso publicado pela ADA em 2019, em que foram analisadas diversas meta-análises, estudos de coorte, estudos observacionais e ensaio clínicos randomizados, estabeleceu-se uma definição para low carb como sendo uma dieta contendo entre 26 e 50\% do valor energético proveniente de carboidratos, e very low carb aquelas contendo de 20 a 50 g/dia de carboidratos ou menos que $26 \%$ do valor energético derivado de carboidratos. ${ }^{11,50-52}$ No presente trabalho, entretanto, diversos autores classificaram como LCD dietas com maiores teores desse macronutriente. ${ }^{17,18,23,28,33,35}$ Diante disso, ainda que se constatem benefícios na adoção de dietas com teores reduzidos de carboidratos por portadores de DM, existe a dificuldade de se estabelecer qual a porcentagem mais adequada de restrição do macronutriente que seja suficiente para que se observem as melhorias causadas pela estratégia alimentar e que também resulte em maior adesão dos pacientes.

Para mensurar os possíveis efeitos das dietas aplicadas aos grupos intervenção, os autores dos artigos eleitos se basearam em parâmetros dos quais se destacaram: alteração de peso e IMC, Hb1Ac, variabilidade ou controle glicêmico, perfil lipídico e alteração nas doses de insulina ou medicação utilizadas. Dentre os 19 
estudos, em quatro não foram observadas vantagens significativas de uma dieta restrita em teores de carboidratos quando comparadas a outras dietas indicadas para o manejo do DM. Contudo, em 15 estudos foram observadas melhoras em um ou mais parâmetros dentre os acima citados.

A melhoria no controle glicêmico e nas concentrações de Hb1Ac é atribuída, pelos autores, ao fato de que indivíduos em uma dieta restrita em carboidratos conseguem manter menor variabilidade nas concentrações de glicose sanguínea. 17,19,20,21,24,25,27,31 Por consequência, isso reduz a necessidade de utilização da farmacologia adjuvante no tratamento do DM. ${ }^{17,19}$

Ainda em suas discussões, os autores relacionam a observação da redução de peso e IMC à menor ingestão calórica, em virtude do efeito sacietogênico que dietas low carb provocam; e a melhoria do perfil lipídico como decorrência de aumento no consumo total de gorduras insaturadas e redução dos níveis de

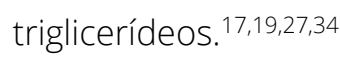

Tais resultados podem representar uma grande vantagem na adoção desta estratégia alimentar no manejo do DM, principalmente na prevenção de complicações advindas de um mal controle da doença. ${ }^{53}$ Dentre as complicações, estão principalmente os distúrbios microvasculares e macrovasculares, que resultam em retinopatia, nefropatia, neuropatia, doença coronariana, doença cerebrovascular, doença arterial periférica; agravos no sistema musculoesquelético, no sistema gastrointestinal, na função cognitiva e na saúde mental, além de ser relacionado a alguns tipos de câncer..$^{53}$

Pouca atenção tem sido dispensada às tendências globais das complicações do DM e ao modo como as características da morbidade associada a essa patologia têm mudado. Dados de morbidade relacionados às doenças secundárias ao DM por vezes mascaram o real problema e a grande necessidade de melhorias nos esforços terapêuticos voltados para essa patologia. ${ }^{53}$

Corroborando tais achados, Feinman e colegas ${ }^{54}$ apresentam 12 pontos de evidência que dão suporte ao uso de dietas pobres em carboidratos como primeira abordagem no tratamento do DM 2, e como o mais eficaz adjuvante à farmacologia no DM 1. Dentre eles, os autores demonstram que como a hiperglicemia é a característica mais marcante do DM, a restrição de carboidratos na dieta tem maior efeito na diminuição das concentrações de glicose no sangue. Também afirmam que durante as epidemias de obesidade e DM 2, os aumentos calóricos que ocasionam essas duas condições estão quase inteiramente relacionados ao aumento de carboidratos na dieta. Os autores também apontam evidências literárias dos benefícios e segurança de dietas LCHP, bem como a falta de evidências que correlacionem o consumo de gorduras totais e saturadas com risco de doença cardiovascular. ${ }^{55-58}$

Apesar disso, ainda existem questionamentos sobre a possibilidade de efeitos colaterais dessas dietas, como o caso de estratégias nas quais a proporção de proteínas da dieta está aumentada em relação às recomendações e aos outros macronutrientes.

As principais críticas de alguns autores surgem em face de seus possíveis danos renais, causados pelo excesso de excreção de nitrogênio durante o metabolismo de proteínas, e que podem causar aumento na pressão glomerular e hiperfiltração. ${ }^{59}$ Segundo os mesmos autores, apesar disso, ainda existem muitos trabalhos que apontam para uma adaptação morfológica do organismo de indivíduos com a função renal normal, tendo em vista o aumento no consumo proteico sem qualquer efeito negativo. ${ }^{59}$ Dentre os estudos reunidos pelo presente trabalho, não foram citados quaisquer tipos de alterações renais como efeito colateral das dietas LCHP.

Encontram-se na literatura autores que chamaram a atenção para outro possível efeito adverso das dietas LC, a respeito dos riscos de se aumentar a proporção de lipídios na dieta em dietas como LCHF. 60-62 Tal 
risco seria proveniente do fato de que, quando a gordura vira a principal fonte de energia da dieta, isso pode resultar em uma proporção muito aumentada de gordura saturada, e levar a uma alteração desfavorável no perfil lipídico, com consequente aumento no risco cardiovascular. Contudo, no presente estudo, existem artigos que demonstram o contrário em seus resultados, nos quais uma dieta restrita em carboidratos resultou em melhora de perfil lipídico. 17,19,27 Esses autores alegam que em uma dieta equilibrada e bem orientada, o consumo de lipídios seguirá um perfil saudável e haverá equilíbrio entre o consumo de gorduras saturadas, monoinsaturadas e poli-insaturadas, bem como aumento no consumo de ômega 3.

As evidências apresentadas pelos estudos selecionados para esta revisão apontam, em sua maioria, benefícios no uso de dietas restritas em carboidratos para o manejo do DM, ou pelo menos uma equivalência quando comparadas a outras estratégias (sem efeitos adversos). Embora todos os estudos selecionados sejam randomizados, um item que, apesar de importante, não foi verificado em nenhum dos trabalhos, é o cegamento, no inglês blinding ou masking.

Esse fato pode ser justificado pelo tipo de estudo escolhido para integrar a presente revisão, que tem como parte imprescindível de sua metodologia que os pesquisadores recebam treinamento para a aplicação da intervenção e, portanto, tenham conhecimento sobre a condição experimental. Assim sendo, a intervenção do tipo duplo-cego (double-blind) fica inviabilizada. Ainda assim, pode-se observar em alguns dos estudos selecionados que os participantes puderam ser cegados quanto ao grupo em que iriam ser alocados intervenção ou controle -, o que diminui o risco de viés nos resultados.

\section{CONCLUSÃO}

De um modo geral, o que se pode inferir da maioria dos estudos nos quais foram propostas intervenções dietoterápicas utilizando dietas com baixos teores de carboidratos e altos teores dos outros macronutrientes, sejam gorduras ou proteínas, é que essa abordagem nutricional tende a garantir melhor controle do DM, tanto no tipo 1 quanto no 2. E ainda, na maioria dos casos, as intervenções não apresentaram eventos adversos não manejáveis.

Tais deduções são plausíveis, considerando os efeitos dominantes dos carboidratos da dieta na glicemia pós-prandial, no controle glicêmico geral e nas concentrações de Hb1Ac, bem como nas consequentes reduções nas doses de insulina e medicações requeridas em uma dieta LC. Os resultados, confirmados em muitos ensaios clínicos, indicam melhoras em parâmetros capazes de prevenir as complicações crônicas do DM.

Contudo, à luz das limitações dos estudos, que ainda não são numerosos - no caso do DM 1 - e precisam ter suas hipóteses verificadas em longo prazo, essas descobertas por si só ainda não foram suficientes para configurar uma estratégia oficial no controle do DM, recomendada pelas principais diretrizes que legislam nessa área. Pesquisas adicionais devem continuar a ser realizadas, a fim de determinar o grau de restrição de carboidratos (e outros aspectos da dieta) necessários para alcançar esses benefícios, um regime ótimo de insulina e/ou medicação para acompanhar essa estratégia (especificamente, para evitar hipoglicemia severa), e para garantir segurança e eficácia no tratamento, prevenindo as complicações e diminuindo a morbidade do DM. 


\section{REFERÊNCIAS}

1. American Diabetes Association (US). Summary of Revisions for the 2009 Clinical Practice Recommendations. Diabetes Care. 2009;32(Suppl 1):S3-5. DOI https://doi.org/10.2337/dc09-S003

2. Spitz AF, Kanani H. Change in HbA1c as a measure of quality of diabetes care. Diabetes Care. 2006;29(5):1183-4. DOI https://doi.org/10.2337/dc05-2032

3. Dias VM, Pandini JÁ, Nunes RR, Sperandei SLM, Portella ES, Cobas RA, et al. Influência do índice glicêmico da dieta sobre parâmetros antropométricos e bioquímicos em pacientes com diabetes tipo 1. Arq Bras Endocrinol Metab. 2010;54(9):801-6. DOI https://doi.org/10.1590/S0004-27302010000900005

4. Miller KM, Foster NC, Beck, RW, Bergenstal RM, DuBose SN, DiMeglio LA, et al. Current state of type 1 diabetes treatment in the US: updated data from the T1D Exchange clinic registry. Diabetes Care. 2015;38(6) 971-978. DOI https://doi.org/10.2337/dc15-0078

5. Rohlfing CL, Wiedmeyer HM, Little RR, England JD, Tennill A, Goldstein DE. Defining the relationship between plasma glucose and HbA1c: analysis of glucose profiles and HbA1c in the Diabetes Control and Complications Trial. Diabetes Care. 2002;25(2):275-278. DOI https://doi.org/10.2337/diacare.25.2.275

6. Sociedade Brasileira de Diabetes. Diretrizes da Sociedade Brasileira de Diabetes 2017-2018. São Paulo: Editora Clannad; 2017.

7. Rocha FM. Dieta baixa em carboidratos para o tratamento de diabetes tipo 2 [monografia]. Brasília: Centro Universitário de Brasília UniCEUB, Curso de Nutrição, 2016.

8. Nielsen JV, Joensson EA. Low-carbohydrate diet in type 2 diabetes: stable improvement of bodyweight and glycemic control during 44 months follow-up. Nutr Metab. 2008;5(1):14. DOI https://doi.org/10.1186/1743-7075-5-14

9. Guldbrand H, Dizdar B, Bunjaku B, Lindström T, Bachrach-Lindström M, Fredrikson M, et al. In type 2 diabetes, randomisation to advice to follow a low-carbohydrate diet transiently improves glycaemic control compared with advice to follow a low-fat diet producing a similar weight loss. Diabetologia. 2012;55(8):2118-2127. DOI https://doi.org/10.1007/s00125-012-2567-4

10. Westman EC, Yancy WS, Mavropoulos JC, Marquart M, McDuffie JR. The effect of a low-carbohydrate, ketogenic diet versus a lowglycemic index diet on glycemic control in type 2 diabetes mellitus. Nutr Metab. 2008;5(1):36. DOI https://doi.org/10.1186/17437075-5-36

11. Evert AB, Dennison M, Gardner CD, Garvey WT, Lau KHK, MacLeod J, et al. Nutrition therapy for adults with diabetes or prediabetes: a consensus report. Diabetes Care. 2019;42(5):731-754. DOI https://doi.org/10.2337/dci19-0014

12. Miller KM, Foster NC, Beck RW, Bergenstal RM, DuBose SN, DiMeglio LA, et al. Current state of type 1 diabetes treatment in the US: updated data from the T1D Exchange clinic registry. Diabetes Care. 2015;38(6):971-978. DOI https://doi.org/10.2337/dc15-0078

13. Lennerz BS, Barton A, Bernstein RK, Dikeman RD, Diulus C, Hallberg S, et al. Management of type 1 diabetes with a very lowcarbohydrate diet. Pediatrics. 2018;141(6):e20173349. DOI https://doi.org/10.1542/peds.2017-3349

14. Moher D, Liberati A, Tetzlaff J, Altman, DG. Preferred reporting items for systematic reviews and meta-analyses: the PRISMA statement. Plos Med. 2009;6(7):e1000097. DOI 10.7326/0003-4819-151-4-200908180-00135

15. Jadad AR, Moore RA, Carroll D, Jenkinson C, Reynolds DJ, Gavaghan DJ, et al. Assessing the quality of reports of randomized clinical trials: is blinding necessary? Controlled Clin Trials. 1996;17(1):1-12. DOI https://doi.org/10.1016/0197-2456(95)00134-4

16. Berger, VW, Alperson, SY. A General Framework for the Evaluation of Clinical Trial Quality. Rev Recent Clin Trials. 2009;4(2):79-88. DOI $10.2174 / 157488709788186021$

17. Wang LL, Wang Q, Hong Y, Ojo O, Jiang Q, Hou YY,et al. The effect of low-carbohydrate diet on glycemic control in patients with Type 2 Diabetes Mellitus. Nutrients. 2018;10(6):661. DOI https://doi.org/10.3390/nu10060661

18. Watson NA, Dyer KA, Buckley, JD, Brinkworth GD, Coates AM, Parfitt G, et al. (2018). Comparison of two low-fat diets, differing in protein and carbohydrate, on psychological wellbeing in adults with obesity and type 2 diabetes: a randomised clinical trial. Nutr J. 2018;17(1):62. DOI 10.1186/s12937-018-0367-5 
19. Tay J, Thompson CH, Luscombe-Marsh ND, Wycherley TP, Noakes M, Buckley JD, et al. Effects of an energy-restricted lowcarbohydrate, high unsaturated fat/low saturated fat diet versus a high-carbohydrate, low-fat diet in type 2 diabetes: A 2-year randomized clinical trial. Diabetes Obes Metab. 2017;20(4):858-871. DOI 10.1111/dom.13164

20. Ranjan A, Schmidt S, Damm-Frydenberg C, Holst JJ, Madsbad S, Nørgaard K. Short-term effects of a low carbohydrate diet on glycaemic variables and cardiovascular risk markers in patients with type 1 diabetes: A randomized open-label crossover trial. Diabetes Obes Metab. 2017;19(10):1479-1484. DOI 10.1111/dom.12953

21. Saslow LR, Daubenmier JJ, Moskowitz JT, Kim S, Murphy EJ, Phinney SD, et al. Twelve-month outcomes of a randomized trial of a moderate-carbohydrate versus very low-carbohydrate diet in overweight adults with type 2 diabetes mellitus or prediabetes. Nutr Diabetes. 2017;7(12):304. DOI https://doi.org/10.1038/s41387-017-0006-9

22. Wycherley TP, Thompson CH, Buckley JD, Luscombe-Marsh ND, Noakes M, Wittert GA, et al. Long-term effects of weight loss with a very-low carbohydrate, low saturated fat diet on flow mediated dilatation in patients with type 2 diabetes: A randomised controlled trial. Atherosclerosis. 2016;252,28-31. DOI 10.1016/j

23. Raygan F, Bahmani F, Kouchaki E, Aghadavod E, Sharifi S, Akbari E, et al. Comparative effects of carbohydrate versus fat restriction on metabolic profiles, biomarkers of inflammation and oxidative stress in overweight patients with Type 2 diabetic and coronary heart disease: A randomized clinical trial. ARYA Ather. 2016;12(6):266. PMID 28607566

24. Sato J, Kanazawa A, Makita S, Hatae C, Komiya K, Shimizu T, et al. A randomized controlled trial of 130 g/day low-carbohydrate diet in type 2 diabetes with poor glycemic control. Clin Nutr. 2016;36(4):992-1000. DOI 10.1016/j.clnu.2016.07.003

25. Krebs JD, Strong AP, Cresswell P, Reynolds AN, Hanna A, Haeusler S. A randomised trial of the feasibility of a low carbohydrate diet vs standard carbohydrate counting in adults with type 1 diabetes taking body weight into account. Asia Pac J Clin Nutr. 2016;25(1):7884. DOI 10.6133/apjen.2016.25.1.11

26. Brinkworth GD, Luscombe-Marsh ND, Thompson CH, Noakes M, Buckley JD, Wittert G, et al. Long-term effects of very lowcarbohydrate and high-carbohydrate weight-loss diets on psychological health in obese adults with type 2 diabetes: randomized controlled trial. J Intern Med. 2016;280(4):388-397. DOI 10.1111/joim.12501

27. Tay J, Luscombe-Marsh ND, Thompson CH, Noakes M, Buckley JD, Wittert GA, et al. Comparison of low-and high-carbohydrate diets for type 2 diabetes management: a randomized trial. Am J Clin Nutr. 2015;102(4):780-790. DOI 10.3945/ajcn.115.112581

28. Rock CL, Flatt SW, Pakiz B, Taylor KS, Leone AF, Brelje K, et al. Weight loss, glycemic control, and cardiovascular disease risk factors in response to differential diet composition in a weight loss program in type 2 diabetes: a randomized controlled trial. Diabetes Care. 2014;37(6):1573-1580. DOI 10.2337/dc13-2900

29. Jonasson L, Guldbrand H, Lundberg AK, Nystrom FH. Advice to follow a low-carbohydrate diet has a favourable impact on low-grade inflammation in type 2 diabetes compared with advice to follow a low-fat diet. Ann Med. 2014;46(3):182-187. DOI $10.3109 / 07853890.2014 .894286$

30. Guldbrand H, Lindström T, Dizdar B, Bunjaku B, Östgren CJ, Nystrom FH, et al. Randomization to a low-carbohydrate diet advice improves health related quality of life compared with a low-fat diet at similar weight-loss in Type 2 diabetes mellitus. Diabetes Res Clin Pract. 2014;106(2):221-227. DOI 10.1016/j.diabres.2014.08.032

31. Saslow LR, Kim S, Daubenmier JJ, Moskowitz JT, Phinney SD, Goldman V, et al. A randomized pilot trial of a moderate carbohydrate diet compared to a very low carbohydrate diet in overweight or obese individuals with type 2 diabetes mellitus or prediabetes. Plos One. 2014;9(4):e91027. DOl 10.1371/journal.pone.0091027

32. Goldstein T, Kark JD, Berry EM, Adler B, Ziv E, Raz I. The effect of a low carbohydrate energy-unrestricted diet on weight loss in obese type 2 diabetes patients-a randomized controlled trial. e-SPEN, E Spen Eur E J Clin Nutr Metab. 2011;6(4):e178-e186. DOI https://doi.org/10.1016/j.eclnm.2011.04.003

33. Larsen RN, Mann NJ, Maclean E, Shaw JE. The effect of high-protein, low-carbohydrate diets in the treatment of type 2 diabetes: a 12 month randomised controlled trial. Diabetologia. 2011;54(4):731-740. DOI 10.1007/s00125-010-2027-y 
34. Davis NJ, Tomuta N, Schechter C, Isasi CR, Segal-Isaacson CJ, Stein D, et al. Comparative study of the effects of a 1-year dietary intervention of a low-carbohydrate diet versus a low-fat diet on weight and glycemic control in type 2 diabetes. Diabetes Care. 2009;32(7):1147-1152. DOI 10.2337/dc08-2108

35. Wolever TM, Gibbs AL, Mehling C, Chiasson JL, Connelly PW, Josse RG et al. The Canadian Trial of Carbohydrates in Diabetes (CCD), a 1-y controlled trial of low-glycemic-index dietary carbohydrate in type 2 diabetes: no effect on glycated hemoglobin but reduction in C-reactive protein. A m J Clin Nutr. 2008;87(1):114-125. DOI 10.3410/f.1108481.564545

36. Turton JL, Raab R, Rooney KB. Low-carbohydrate diets for type 1 diabetes mellitus: A systematic review. Plos One 2018;13(3):e0194987. DOI 10.1371/journal.pone.0194987

37. Brazeau AS, Mircescu H, Desjardins K, Leroux C, Strychar I, Ekoé JM, et al. Carbohydrate counting accuracy and blood glucose variability in adults with type 1 diabetes. Diabetes Res Clin Pract.2013;99(1):19-23. DOI 10.1016/j.diabres.2012.10.024

38. Lawton J, Rankin D, Cooke DD, Clark M, Elliot J, Heller S, et al. Dose adjustment for normal eating: a qualitative longitudinal exploration of the food and eating practices of type 1 diabetes patients converted to flexible intensive insulin therapy in the UK. Diabetes Res Clin Pract. 2011;91(1):87-93. DOI 10.1016/j.diabres.2010.11.007

39. Mehta SN, Quinn N, Volkening LK, Laffel L. Impact of carbohydrate counting on glycemic control in children with type 1 diabetes. Diabetes Care. 2009;32(6):1014-1016. DOI 10.2337/dc08-2068

40. Smart CE, Ross K, Edge JA, King BR, McElduff P, Collins CE. Can children with type 1 diabetes and their caregivers estimate the carbohydrate content of meals and snacks? Diabetic Med. 2010;27(3):348-353. DOI 10.1111/j.1464-5491.2010.02945.x

41. Gingras V, Rabasa-Lhoret R, Messier V, Ladouceur M, Legault L, Haidar A. Efficacy of dual-hormone artificial pancreas to alleviate the carbohydrate-counting burden of type 1 diabetes: A randomized crossover trial. Diabetes Metab. 2016;42(1):47-54. DOI 10.1016/j.diabet.2015.05.001

42. Castañeda-González LM, Bacardi Gascon M, Jimenez Cruz A. Effects of low carbohydrate diets on weight and glycemic control among type 2 diabetes individuals: a systemic review of RCT greater than 12 weeks. Nutr Hosp. 2011;26(6). DOI 10.1590/S021216112011000600013

43. Forte LB, Fernandes VO, de Queiroz PC, de Carvalho MMD, Gadelha DD, Junior RMM. Hemoglobina glicada A1c no diabetes. Revista de Medicina da UFC. 2019;59(1):79-80. DOI https://doi.org/10.20513/2447-6595.2019v59n1p79-80

44. Brouns F. (2018). Overweight and diabetes prevention: is a low-carbohydrate-high-fat diet recommendable?. Eur J Nutr. 2018;57(4):1301-1312. DOI 10.1007/s00394-018-1636-y

45. Noakes TD, Windt J. Evidence that supports the prescription of low-carbohydrate high-fat diets: a narrative review. Br J Sports Med. 2017;51(2):133-139. DOI http://dx.doi.org/10.1136/bjsports-2016-096491

46. Daly ME, Paisey R, Paisey R, Millward BA, Eccles C, Williams K, et al. Short-term effects of severe dietary carbohydrate-restriction advice in Type 2 diabetes_a randomized controlled trial. Diabetic Med. 2006;23(1):15-20. DOI 10.1111/j.1464-5491.2005.01760.x

47. Gardner CD, Kiazand A, Alhassan S, Kim S, Stafford RS, Balise RR et al. Comparison of the Atkins, Zone, Ornish, and LEARN diets for change in weight and related risk factors among overweight premenopausal women: the A TO Z Weight Loss Study: a randomized trial. JAMA. 2007;297(9):969-977. DOI 10.1001/jama.297.9.969

48. Krebs NF, Gao D, Gralla J, Collins JS, Johnson SL. Efficacy and safety of a high protein, low carbohydrate diet for weight loss in severely obese adolescents. J Pediatr. 2010;157(2):252-258. DOI 10.1016/j.jpeds.2010.02.010

49. Hession M, Rolland C, Kulkarni U, Wise A, Broom J. Systematic review of randomized controlled trials of low-carbohydrate vs. lowfat/low-calorie diets in the management of obesity and its comorbidities. Obes Rev. 2009;10(1):36-50. DOI https://doi.org/10.1111/j.1467-789X.2008.00518.x

50. Van Zuuren EJ, Fedorowicz Z, Kuijpers T, Pijl H. Effects of low-carbohydrate- compared with low-fat-diet interventions on metabolic control in people with type 2 diabetes: a systematic review including GRADE assessments. Am J Clin Nutr. 2018;108:300-331. DOI 10.1093/ajen/nqy096 
51. Sainsbury E, Kizirian NV, Partridge SR, Gill T, Colagiuri S, Gibson AA. Effect of dietary carbohydrate restriction on glycemic control in adults with diabetes: a systematic review and metaanalysis. Diabetes Res Clin Pract 2018;139:239-252. DOI 10.1016/j.diabres.2018.02.026

52. Snorgaard O, Poulsen GM, Andersen HK, Astrup A. Systematic review and meta-analysis of dietary carbohydrate restriction in patients with type 2 diabetes. BMJ Open Diabetes Res Care. 2017;5:e000354. DOI http://dx.doi.org/10.1136/bmjdrc-2016-000354

53. Gregg EW, Sattar N, Ali MK. The changing face of diabetes complications. Lancet Diabetes Endocrinol. 2016;4(6):537-547. DOI 10.1016/S2213-8587(16)30010-9

54. Feinman RD, Pogozelski WK, Astrup A, Bernstein RK, Fine EJ, Westman EC, et al. Dietary carbohydrate restriction as the first approach in diabetes management: critical review and evidence base. Nutrition. 2015;31(1):1-13. DOI 10.1016/j.nut.2014.06.011

55. Siri-Tarino PW, Sun Q, Hu FB, Krauss RM. Saturated fat, carbohydrate, and cardiovascular disease. Am J Clin Nutr. 2010;91:502-9. DOI 10.3945/ajcn.2008.26285

56. Weinberg SL. The diet-heart hypothesis: a critique. J Am Coll Cardiol. 2004;43:731-3. DOI 10.1016/j.jacc.2003.10.034

57. Teicholz N. The big fat surprise. Why butter, meat \& cheese belong in a healthy diet. New York: Simon \& Schuster; 2014.

58. Jakobsen MU, O'Reilly EJ, Heitmann BL, Pereira MA, Balter K, Fraser GE, et al. Major types of dietary fat and risk of coronary heart disease: a pooled analysis of 11 cohort studies. Am J Clin Nutr. 2009;89:1425-32. DOI 10.3945/ajcn.2008.27124

59. Paoli A, Rubini A, Volek JS, Grimaldi KA. Beyond weight loss: a review of the therapeutic uses of very-low-carbohydrate (ketogenic) diets. Eur J Clin Nutr. 2013;67(8):789. DOI 10.1038/ejcn.2013.116

60. Michaliszyn SF, Shaibi GQ, Quinn L, Fritschi C, Faulkner MS. Physical fitness, dietary intake, and metabolic control in adolescents with type 1 diabetes. Pediatr Diabetes. 2009;10(6):389-394. DOI 10.1111/j.1399-5448.2009.00500.x

61. Leow ZZX, Guelfi KJ, Davis EA, Jones TW, Fournier PA. The glycaemic benefits of a very-low-carbohydrate ketogenic diet in adults with Type 1 diabetes mellitus may be opposed by increased hypoglycaemia risk and dyslipidaemia. Diabetic Med. 2018;35(9):1258-1263. DOI 10.1111/dme.13663

62. Bock M, Lobley K, Anderson D, Davis E, Donaghue K, Pappas M, et al. Endocrine and metabolic consequences due to restrictive carbohydrate diets in children with type 1 diabetes: An illustrative case series. Pediatr Diabetes. 2018;19(1):129-137. DOI 10.1111/pedi.12527

\section{Colaboradoras}

Campos LP e Lobo LMC participaram de todas as etapas, desde a concepção do estudo até a revisão da versão final do artigo.

Conflito de Interesses: as autoras declaram não haver conflito de interesses.

Recebido: 25 de junho de 2019

Aprovado: 05 de janeiro de 2020 